\title{
Optimal stabilization using Lyapunov measures
}

\author{
Arvind Raghunathan Umesh Vaidya
}

\begin{abstract}
Numerical solutions for the optimal feedback stabilization of discrete time dynamical systems is the focus of this paper. Set-theoretic notion of almost everywhere stability introduced by the Lyapunov measure, weaker than conventional Lyapunov function-based stabilization methods, is used for optimal stabilization. The linear PerronFrobenius transfer operator is used to pose the optimal stabilization problem as an infinite dimensional linear program. Set-oriented numerical methods are used to obtain the finite dimensional approximation of the linear program. We provide conditions for the existence of stabilizing feedback controls and show the optimal stabilizing feedback control can be obtained as a solution of a finite dimensional linear program. The approach is demonstrated on stabilization of period two orbit in a controlled standard map.
\end{abstract}

\section{Index Terms}

Almost everywhere stability, optimal stabilization, numerical methods.

\section{INTRODUCTION}

Stability analysis and stabilization of nonlinear systems are two of the most important, extensively studied problems in control theory. Lyapunov functions are used for stability analysis and control Lyapunov functions (CLF) are used in the design of stabilizing feedback controllers. Under the assumption of detectability and stabilizability of the nonlinear system, a positive valued optimal cost function of an optimal control problem (OCP) can also be used as a control Lyapunov function. The optimal controls of OCP are obtained as the solution of the Hamilton Jacobi Bellman (HJB) equation. The HJB equation is a nonlinear partial differential equation and one must resort to approximate numerical schemes for its solution. Numerical schemes typically discretize the state-space; hence, the resulting problem size grows exponentially with the dimension of the state-space. This is commonly referred to as the curse of dimensionality. The approach is particularly attractive for feedback control of nonlinear systems with lower dimensional state space. The method proposed in this paper also suffers from the same drawback. Among the vast literature available on the topic of solving the HJB equation, we briefly review some of the related literature.

Vinter [1] was the first to propose a linear programming approach for nonlinear optimal control of continuous time systems. This was exploited to develop a numerical algorithm, based on semidefinite programming and density function-based formulation by Rantzer and co-workers in [2], [3]. Global almost everywhere stability of stochastic

\footnotetext{
A. Raghunathan is with Mitsubishi Electric Research Laboratories in Cambridge MA 02139 raghunathan@merl.com

U. Vaidya is with the Department of Electrical \& Computer Engineering, Iowa State University, Ames, IA 50011 ugvaidyaeiastate.edu
} 
systems was studied by van Handel [4], using the density function. Lasserre, Hernández-Lerma, and co-workers [5], [6] formulated the control of Markov processes as a solution of the HJB equation. An adaptive space discretization approach is used in [7]; a cell mapping approach is used in [8] and [9], [10] utilizes set oriented numerical methods to convert the HJB to one of finding the minimum cost path on a graph derived from transition. In [11], [12], [13], solutions to stochastic and deterministic optimal control problems are proposed, using a linear programming approach or using a sequence of LMI relaxations. Our paper also draws some connection to research on optimization and stabilization of controlled Markov chains discussed in [14]. Computational techniques based on the viscosity solution of the HJB equation is proposed for the approximation of value function and optimal controls in [15] (Chapter VI).

Our proposed method, in particular the computational approach, draws some similarity with the above discussed references on the approximation of the solution of the HJB equation [8], [9], [10], [15]. Our method, too, relies on discretization of state space to obtain globally optimal stabilizing control. However, our proposed approach differs from the above references in the following two fundamental ways. The first main difference arises due to adoption of non-classical weaker set-theoretic notion of almost everywhere stability for optimal stabilization. This weaker notion of stability allows for the existence of unstable dynamics in the complement of the stabilized attractor set; whereas, such unstable dynamics are not allowed using the classical notion of Lyapunov stability adopted in the above references. This weaker notion of stability is advantageous from the point of view of feedback control design. The notion of almost everywhere stability and density function for its verification was introduced by Rantzer in [16]. Furthermore, Rantzer proved, that unlike the control Lyapunov function, the co-design problem of jointly finding the density function and the stabilizing controller is convex [17]. The Lyapunov measure used in this paper for optimal stabilization can be viewed as a measure corresponding to the density function [18], [19]. Hence, it enjoys the same convexity property for the controller design. This convexity property, combined with the proposed linear transfer operator framework, is precisely exploited in the development of linear programming-based computational framework for optimal stabilization using Lyapunov measures. The second main difference compared to references [14] and [15] is in the use of the discount factor $\gamma>1$ in the cost function (refer to Remark 9]. The discount factor plays an important role in controlling the effect of finite dimensional discretization or the approximation process on the true solution. In particular, by allowing for the discount factor, $\gamma$, to be greater than one, it is possible to ensure that the control obtained using the finite dimensional approximation is truly stabilizing the nonlinear system [20], [21].

In a previous work [20] involving Vaidya, the problem of designing deterministic feedback controllers for stabilization via control Lyapunov measure was addressed. The authors proposed solving the problem by using a mixed integer formulation or a non-convex nonlinear program, which are not computationally efficient. There are two main contributions of this paper. First, we show a deterministic stabilizing feedback controller can be constructed using a computationally cheap tree-growing algorithm (Algorithm 1, Lemma 11. The second main contribution of this paper is the extension of the Lyapunov measure framework introduced in [18] to design optimal stabilization of an attractor set. We prove the optimal stabilizing controllers can be obtained as the solution to a 
linear program. Unlike the approach proposed in [20], the solution to the linear program is guaranteed to yield deterministic controls. This paper is an extended version of the paper that appeared in the 2008 American Control Conference [22].

This paper is organized as follows. In Section [I] we provide a brief overview of key results from [18] and [20] for stability analysis, and stabilization of nonlinear systems using the Lyapunov measure. The transfer operators-based framework is used to formulate the OCP as an infinite dimensional linear program in Section III] A computational approach, based on set-oriented numerical methods, is proposed for the finite dimensional approximation of the linear program in Section IV Simulation results are presented in Section $\mathrm{V}$, followed by conclusions in Section $\mathrm{VI}$

\section{LYAPUNOV MEASURE, STABILITY AND STABILIZATION}

The Lyapunov measure and control Lyapunov measure were introduced in [18], [20] for stability analysis and stabilizing controller design in discrete-time dynamical systems of the form,

$$
x_{n+1}=F\left(x_{n}\right),
$$

where $F: X \rightarrow X$ is assumed to be continuous with $X \subset \mathbb{R}^{q}$, a compact set. We denote by $\mathcal{B}(X)$ the Borel- $\sigma$ algebra on $X$ and $\mathcal{M}(X)$, the vector space of a real valued measure on $\mathcal{B}(X)$. The mapping, $F$, is assumed to be nonsingular with respect to the Lebesgue measure $\ell$, i.e., $\ell\left(F^{-1}(B)\right)=0$, for all sets $B \in \mathcal{B}(X)$, such that $\ell(B)=0$. In this paper, we are interested in optimal stabilization of an attractor set defined as follows:

Definition 1 (Attractor set): A set $\mathcal{A} \subset X$ is said to be forward invariant under $F$, if $F(\mathcal{A})=\mathcal{A}$. A closed forward invariant set, $\mathcal{A}$, is said to be an attractor set, if it there exists a neighborhood $V \subset X$ of $\mathcal{A}$, such that $\omega(x) \subset \mathcal{A}$ for all $x \in V$, where $\omega(x)$ is the $\omega$ limit set of $x$ [18].

Remark 2: In the following definitions and theorems, we will use the notation, $U(\epsilon)$, to denote the $\epsilon>0$ neighborhood of the attractor set $\mathcal{A}$ and $m \in \mathcal{M}(X)$, a finite measure absolutely continuous with respect to Lebesgue.

Definition 3 (Almost everywhere stable with geometric decay): The attractor set $\mathcal{A} \subset X$ for a dynamical system (1) is said to be almost everywhere (a.e.) stable with geometric decay with respect to some finite measure, $m \in$ $\mathcal{M}(X)$, if given any $\epsilon>0$, there exists $M(\epsilon)<\infty$ and $\beta<1$, such that $m\left\{x \in \mathcal{A}^{c}: F^{n}(x) \in X \backslash U(\epsilon)\right\}<M(\epsilon) \beta^{n}$.

The above set-theoretic notion of a.e. stability is introduced in [18] and verified by using the linear transfer operator framework. For the discrete time dynamical system (1), the linear transfer Perron Frobenius (P-F) operator [23] denoted by $\mathbb{P}_{F}: \mathcal{M}(X) \rightarrow \mathcal{M}(X)$ is given by,

$$
\left[\mathbb{P}_{F} \mu\right](B)=\int_{X} \chi_{B}(F(x)) d \mu(x)=\mu\left(F^{-1}(B)\right),
$$

where $\chi_{B}(x)$ is the indicator function supported on the set $B \in \mathcal{B}(X)$ and $F^{-1}(B)$ is the inverse image of set $B$. We define a sub-stochastic operator as a restriction of the P-F operator on the complement of the attractor set as follows:

$$
\left[\mathbb{P}_{F}^{1} \mu\right](B):=\int_{\mathcal{A}^{c}} \chi_{B}(F(x)) d \mu(x)
$$


for any set $B \in \mathcal{B}\left(\mathcal{A}^{c}\right)$ and $\mu \in \mathcal{M}\left(\mathcal{A}^{c}\right)$. The condition for the a.e. stability of an attractor set $\mathcal{A}$ with respect to some finite measure $m$ is defined in terms of the existence of the Lyapunov measure $\bar{\mu}$, defined as follows [18]

Definition 4 (Lyapunov measure): The Lyapunov measure is defined as any non-negative measure $\bar{\mu}$, finite outside $U(\epsilon)$ (see Remark 2), and satisfies the following inequality, $\left[\mathbb{P}_{F}^{1} \bar{\mu}\right](B)<\gamma^{-1} \bar{\mu}(B)$, for some $\gamma \geq 1$ and all sets $B \in \mathcal{B}(X \backslash U(\epsilon))$, such that $m(B)>0$.

The following theorem from [24] provides the condition for a.e. stability with geometric decay.

Theorem 5: An attractor set $\mathcal{A}$ for the dynamical system (1) is a.e. stable with geometric decay with respect to finite measure $m$, if and only if for all $\epsilon>0$ there exists a non-negative measure $\bar{\mu}$, which is finite on $\mathcal{B}(X \backslash U(\epsilon))$ and satisfies

$$
\gamma\left[\mathbb{P}_{F}^{1} \bar{\mu}\right](B)-\bar{\mu}(B)=-m(B)
$$

for all measurable sets $B \subset X \backslash U(\epsilon)$ and for some $\gamma>1$.

Proof: We refer readers to Theorem 5 from [21] for the proof.

We consider the stabilization of dynamical systems of the form $x_{n+1}=T\left(x_{n}, u_{n}\right)$, where $x_{n} \in X \subset \mathbb{R}^{q}$ and $u_{n} \in U \subset \mathbb{R}^{d}$ are the state and the control input, respectively. Both $X$ and $U$ are assumed compact. The objective is to design a feedback controller, $u_{n}=K\left(x_{n}\right)$, to stabilize the attractor set $\mathcal{A}$. The stabilization problem is solved using the Lyapunov measure by extending the P-F operator formalism to the control dynamical system [20]. We define the feedback control mapping $C: X \rightarrow Y:=X \times U$ as $C(x)=(x, K(x))$. We denote by $\mathcal{B}(Y)$ the Borel- $\sigma$ algebra on $Y$ and $\mathcal{M}(Y)$ the vector space of real valued measures on $\mathcal{B}(Y)$. For any $\mu \in \mathcal{M}(X)$, the control mapping $C$ can be used to define a measure, $\theta \in \mathcal{M}(Y)$, as follows:

$$
\begin{aligned}
& \theta(D):=\left[\mathbb{P}_{C} \mu\right](D)=\mu\left(C^{-1}(D)\right) \\
& {\left[\mathbb{P}_{C^{-1}} \theta\right](B):=\mu(B)=\theta(C(B)),}
\end{aligned}
$$

for all sets $D \in \mathcal{B}(Y)$ and $B \in \mathcal{B}(X)$. Since $C$ is an injective function with $\theta$ satisfying [5], it follows from the theorem on disintegration of measure [25] (Theorem 5.8) there exists a unique disintegration $\theta_{x}$ of the measure $\theta$ for $\mu$ almost all $x \in X$, such that $\int_{Y} f(y) d \theta(y)=\int_{X} \int_{C(x)} f(y) d \theta_{x}(y) d \mu(x)$, for any Borelmeasurable function $f: Y \rightarrow \mathbb{R}$. In particular, for $f(y)=\chi_{D}(y)$, the indicator function for the set $D$, we obtain $\theta(D)=\int_{X} \int_{C(x)} \chi_{D}(y) d \theta_{x}(y) d \mu(x)=\left[\mathbb{P}_{C} \mu\right](D)$. Using the definition of the feedback controller mapping $C$, we write the feedback control system as $x_{n+1}=T\left(x_{n}, K\left(x_{n}\right)\right)=T \circ C\left(x_{n}\right)$. The system mapping $T: Y \rightarrow X$ can be associated with P-F operators $\mathbb{P}_{T}: \mathcal{M}(Y) \rightarrow \mathcal{M}(X)$ as $\left[\mathbb{P}_{T} \theta\right](B)=\int_{Y} \chi_{B}(T(y)) d \theta(y)$. The P-F operator for the composition $T \circ C: X \rightarrow X$ can be written as a product of $\mathbb{P}_{T}$ and $\mathbb{P}_{C}$. In particular, we obtain [21]

$$
\begin{aligned}
& {\left[\mathbb{P}_{T \circ C} \mu\right](B)=\int_{Y} \chi_{B}(T(y)) d\left[\mathbb{P}_{C} \mu\right](y) } \\
= & {\left[\mathbb{P}_{T} \mathbb{P}_{C} \mu\right](B)=\int_{X} \int_{C(x)} \chi_{B}(T(y)) d \theta_{x}(y) d \mu(x) . }
\end{aligned}
$$


The P-F operators, $\mathbb{P}_{T}$ and $\mathbb{P}_{\mathbb{C}}$, are used to define their restriction, $\mathbb{P}_{T}^{1}: \mathcal{M}\left(\mathcal{A}^{c} \times U\right) \rightarrow \mathcal{M}\left(\mathcal{A}^{c}\right)$, and $\mathbb{P}_{C}^{1}:$ $\mathcal{M}\left(\mathcal{A}^{c}\right) \rightarrow \mathcal{M}\left(\mathcal{A}^{c} \times U\right)$ to the complement of the attractor set, respectively, in a way similar to Eq. (3). The control Lyapunov measure introduced in [20] is defined as any non-negative measure $\bar{\mu} \in \mathcal{M}\left(\mathcal{A}^{c}\right)$, finite on $\mathcal{B}(X \backslash U(\epsilon))$, such that there exists a control mapping $C$ that satisfies $\left[\mathbb{P}_{T}^{1} \mathbb{P}_{C}^{1} \bar{\mu}\right](B)<\beta \bar{\mu}(B)$, for every set $B \in \mathcal{B}(X \backslash U(\epsilon))$ and $\beta \leq 1$. Stabilization of the attractor set is posed as a co-design problem of jointly obtaining the control Lyapunov measure $\bar{\mu}$ and the control P-F operator $\mathbb{P}_{C}$ or in particular disintegration of measure $\theta$, i.e., $\theta_{x}$. The disintegration measure $\theta_{x}$, which lives on the fiber of $C(x)$, in general, will not be absolutely continuous with respect to Lebesgue. For the deterministic control map, $K(x)$, the conditional measure, $\theta_{x}(u)=\delta(u-K(x))$, the Dirac delta measure. However, for the purpose of computation, we relax this condition. The purpose of this paper and the following sections are to extend the Lyapunov measure-based framework for the optimal stabilization of nonlinear systems. One of the key highlights of this paper is the deterministic finite optimal stabilizing control is obtained as the solution for a finite linear program.

\section{OPTIMAL STABILIZATION}

The objective is to design a feedback controller for the stabilization of the attractor set, $\mathcal{A}$, in a.e. sense, while minimizing a suitable cost function. Consider the following control system,

$$
x_{n+1}=T\left(x_{n}, u_{n}\right)
$$

where $x_{n} \in X \subset \mathbb{R}^{q}$ and $u_{n} \in U \subset \mathbb{R}^{d}$ are state and control input, respectively, and $T: X \times U=: Y \rightarrow X$. Both $X$ and $U$ are assumed compact. We define $X_{1}:=X \backslash U(\epsilon)$.

Assumption 6: We assume there exists a feedback controller mapping $C_{0}(x)=\left(x, K_{0}(x)\right)$, which locally stabilizes the invariant set $\mathcal{A}$, i.e., there exists a neighborhood $V$ of $\mathcal{A}$ such that $T \circ C_{0}(V) \subset V$ and $x_{n} \rightarrow \mathcal{A}$ for all $x_{0} \in V$; moreover $\mathcal{A} \subset U(\epsilon) \subset V$.

Our objective is to construct the optimal stabilizing controller for almost every initial condition starting from $X_{1}$. Let $C_{1}: X_{1} \rightarrow Y$ be the stabilizing control map for $X_{1}$. The control mapping $C: X \rightarrow X \times U$ can be written as follows:

$$
C(x)=\left\{\begin{array}{lll}
C_{0}(x)=\left(x, K_{0}(x)\right) & \text { for } & x \in U(\epsilon) \\
C_{1}(x)=\left(x, K_{1}(x)\right) & \text { for } \quad x \in X_{1} .
\end{array}\right.
$$

Furthermore, we assume the feedback control system $T \circ C: X \rightarrow X$ is non-singular with respect to the Lebesgue measure, $m$. We seek to design the controller mapping, $C(x)=(x, K(x))$, such that the attractor set $\mathcal{A}$ is a.e. stable with geometric decay rate $\beta<1$, while minimizing the following cost function,

$$
\mathcal{C}_{C}(B)=\int_{B} \sum_{n=0}^{\infty} \gamma^{n} G \circ C\left(x_{n}\right) d m(x),
$$

where $x_{0}=x$, the cost function $G: Y \rightarrow \mathbb{R}$ is assumed a continuous non-negative real-valued function, such that

$G(\mathcal{A}, 0)=0, x_{n+1}=T \circ C\left(x_{n}\right)$, and $0<\gamma<\frac{1}{\beta}$. Note, that in the cost function $\sqrt{8}$, $\gamma$ is allowed greater than one and this is one of the main departures from the conventional optimal control problem, where $\gamma \leq 1$. However, 
under the assumption that the controller mapping $C$ renders the attractor set a.e. stable with a geometric decay rate, $\beta<\frac{1}{\gamma}$, the cost function 8 is finite.

Remark 7: To simplify the notation, in the following we will use the notion of the scalar product between continuous function $h \in \mathcal{C}^{0}(X)$ and measure $\mu \in \mathcal{M}(X)$ as $\langle h, \mu\rangle_{X}:=\int_{X} h(x) d \mu(x)$ [23].

The following theorem proves the cost of stabilization of the set $\mathcal{A}$ as given in Eq. [8] can be expressed using the control Lyapunov measure equation.

Theorem 8: Let the controller mapping, $C(x)=(x, K(x))$, be such that the attractor set $\mathcal{A}$ for the feedback control system $T \circ C: X \rightarrow X$ is a.e. stable with geometric decay rate $\beta<1$. Then, the cost function [8] is well defined for $\gamma<\frac{1}{\beta}$ and, furthermore, the cost of stabilization of the attractor set $\mathcal{A}$ with respect to Lebesgue almost every initial condition starting from set $B \in \mathcal{B}\left(X_{1}\right)$ can be expressed as follows:

$$
\begin{gathered}
\mathcal{C}_{C}(B)=\int_{B} \sum_{n=0}^{\infty} \gamma^{n} G \circ C\left(x_{n}\right) d m(x) \\
=\int_{\mathcal{A}^{c} \times U} G(y) d\left[\mathbb{P}_{C}^{1} \bar{\mu}_{B}\right](y)=\left\langle G, \mathbb{P}_{C}^{1} \bar{\mu}_{B}\right\rangle_{\mathcal{A}^{c} \times U},
\end{gathered}
$$

where, $x_{0}=x$ and $\bar{\mu}_{B}$ is the solution of the following control Lyapunov measure equation,

$$
\gamma \mathbb{P}_{T}^{1} \cdot \mathbb{P}_{C}^{1} \bar{\mu}_{B}(D)-\bar{\mu}_{B}(D)=-m_{B}(D)
$$

for all $D \in \mathcal{B}\left(X_{1}\right)$ and where $m_{B}(\cdot):=m(B \cap \cdot)$ is a finite measure supported on the set $B \in \mathcal{B}\left(X_{1}\right)$.

Proof: The proof is omitted in this paper, due to limited space but can be found in the online version of the paper [21] (Theorem 8).

By appropriately selecting the measure on the right-hand side of the control Lyapunov measure equation (10) (i.e., $m_{B}$ ), stabilization of the attractor set with respect to a.e. initial conditions starting from a particular set can be studied. The minimum cost of stabilization is defined as the minimum over all a.e. stabilizing controller mappings, $C$, with a geometric decay as follows:

$$
\mathcal{C}^{*}(B)=\min _{C} \mathcal{C}_{C}(B)
$$

Next, we write the infinite dimensional linear program for the optimal stabilization of the attractor set $\mathcal{A}$. Towards this goal, we first define the projection map, $P_{1}: \mathcal{A}^{c} \times U \rightarrow \mathcal{A}^{c}$ as: $P_{1}(x, u)=x$, and denote the P-F operator corresponding to $P_{1}$ as $\mathbb{P}_{P_{1}}: \mathcal{M}\left(\mathcal{A}^{c} \times U\right) \rightarrow \mathcal{M}\left(\mathcal{A}^{c}\right)$, which can be written as $\left[\mathbb{P}_{P_{1}}^{1} \theta\right](D)=\int_{\mathcal{A}^{c} \times U} \chi_{D}\left(P_{1}(y)\right) d \theta(y)=$ $\int_{D \times U} d \theta(y)=\mu(D)$. Using this definition of projection mapping, $P_{1}$, and the corresponding P-F operator, we can write the linear program for the optimal stabilization of set $B$ with unknown variable $\theta$ as follows:

$$
\min _{\theta \geq 0}\langle G, \theta\rangle_{\mathcal{A}^{c} \times U} \text {, s.t. } \gamma\left[\mathbb{P}_{T}^{1} \theta\right](D)-\left[\mathbb{P}_{P_{1}}^{1} \theta\right](D)=-m_{B}(D),
$$

for $D \in \mathcal{B}\left(X_{1}\right)$.

Remark 9: Observe the geometric decay parameter satisfies $\gamma>1$. This is in contrast to most optimization problems studied in the context of Markov-controlled processes, such as in Lasserre and Hernández-Lerma [5]. Average cost and discounted cost optimality problems are considered in [5], [15]. The additional flexibility provided 
by $\gamma>1$ guarantees the controller obtained from the finite dimensional approximation of the infinite dimensional program (12) also stabilizes the attractor set for system (6). For a more detailed discussion on the role of $\gamma$ on the finite dimensional approximation, we refer readers to the online version of the paper [21].

\section{COMPUTATIONAL APPROACH}

The objective of the present section is to present a computational framework for the solution of the finitedimensional approximation of the optimal stabilization problem in $(12)$. There exists a number of references related to the solution of infinite dimensional linear programs (LPs), in general, and those arising from the control of Markov processes. Some will be described next. The monograph by Anderson and Nash [26] is an excellent reference on the properties of infinite dimensional LPs.

Our intent is to use the finite-dimensional approximation as a tool to obtain stabilizing controls to the infinitedimensional system. First, we will derive conditions under which solutions to the finite-dimensional approximation exist.

Following [18] and [20], we discretize the state-space and control space for the purposes of computations as described below. Borrowing the notation from [20], let $\mathcal{X}_{N}:=\left\{D_{1}, \ldots, D_{i}, \ldots, D_{N}\right\}$ denote a finite partition of the state-space $X \subset \mathbb{R}^{q}$. The measure space associated with $\mathcal{X}_{N}$ is $\mathbb{R}^{N}$. We assume without loss of generality that the attractor set, $\mathcal{A}$, is contained in $D_{N}$, that is, $\mathcal{A} \subseteq D_{N}$. Similarly, the control space, $U$, is quantized and the control input is assumed to take only finitely many control values from the quantized set, $\mathcal{U}_{M}=\left\{u^{1}, \ldots, u^{a}, \ldots, u^{M}\right\}$, where $u^{a} \in \mathbb{R}^{d}$. The partition, $\mathcal{U}_{M}$, is identified with the vector space, $\mathbb{R}^{N \times M}$. The system map that results from choosing the controls $u_{N}$ is denoted as $T_{u_{N}}$ and the corresponding P-F operator is denoted as $P_{T_{u_{N}}} \in \mathbb{R}^{N \times N}$. Fixing the controls on all sets of the partition to $u^{a}$, i.e., $u_{N}\left(D_{i}\right)=u^{a}$, for all $D_{i} \in \mathcal{X}_{N}$, the system map that results is denoted as $T_{a}$ with the corresponding P-F operator denoted as $P_{T_{a}} \in \mathbb{R}^{N \times N}$. The entries for $P_{T_{a}}$ are calculated as: $\left(P_{T_{a}}\right)_{(i j)}:=\frac{m\left(T_{a}^{-1}\left(D_{j}\right) \cap D_{i}\right)}{m\left(D_{i}\right)}$, where $m$ is the Lebesgue measure and $\left(P_{T_{a}}\right)_{(i j)}$ denotes the $(i, j)$-th entry of the matrix. Since $T_{a}: X \rightarrow X$, we have $P_{T_{a}}$ is a Markov matrix. Additionally, $P_{T_{a}}^{1}: \mathbb{R}^{N-1} \rightarrow \mathbb{R}^{N-1}$ will denote the finite dimensional counterpart of the P-F operator restricted to $\mathcal{X}_{N} \backslash D_{N}$, the complement of the attractor set. It is easily seen that $P_{T_{a}}^{1}$ consists of the first $(N-1)$ rows and columns of $P_{T_{a}}$.

In [18] and [20], stability analysis and stabilization of the attractor set are studied, using the above finite dimensional approximation of the P-F operator. The finite dimensional approximation of the P-F operator results in a weaker notion of stability, referred to as coarse stability [18], [21]. Roughly speaking, coarse stability means stability modulo attractor sets with domain of attraction smaller than the size of cells within the partition.

With the above quantization of the control space and partition of the state space, the determination of the control $u(x) \in U$ (or equivalently $K(x)$ ) for all $x \in \mathcal{A}^{c}$ has now been cast as a problem of choosing $u_{N}\left(D_{i}\right) \in \mathcal{U}_{M}$ for all sets $D_{i} \subset \mathcal{X}_{N}$. The finite dimensional approximation of the optimal stabilization problem (12) is equivalent to solving the following finite-dimensional LP:

$$
\min _{\theta^{a}, \mu \geq 0} \sum_{a=1}^{M}\left(G^{a}\right)^{\prime} \theta^{a}, \quad \text { s.t. } \gamma \sum_{a=1}^{M}\left(P_{T_{a}}\right)^{\prime} \theta^{a}-\sum_{a=1}^{M} \theta^{a}=-m,
$$


where we have used the notation $(\cdot)^{\prime}$ for the transpose operation, $m \in \mathbb{R}^{N-1}$ and $(m)_{(j)}>0$ denote the support of Lebesgue measure, $m$, on the set $D_{j}, G^{a} \in \mathbb{R}^{N-1}$ is the cost defined on $\mathcal{X}_{N} \backslash D_{N}$ with $\left(G^{a}\right)_{(j)}$ the cost associated with using control action $u^{a}$ on set $D_{j} ; \theta^{a} \in \mathbb{R}^{N-1}$ are, respectively, the discrete counter-parts of infinite-dimensional measure quantities in (12). In the LP [13], we have not enforced the constraint,

$$
\left(\theta^{a}\right)_{(j)}>0 \text { for exactly one } a \in\{1, \ldots, M\},
$$

for each $j=1, \ldots,(N-1)$. The above constraint ensures the control on each set in unique. We prove in the following the uniqueness can be ensured without enforcing the constraint, provided the LP (13) has a solution. To this end, we introduce the dual LP associated with the LP in (13). The dual to the LP in 13) is,

$$
\max _{V} m^{\prime} V \text {, s.t. } V \leq \gamma P_{T_{a}}^{1} V+G^{a} \forall a=1, \ldots, M .
$$

In the above LP (15), $V$ is the dual variable to the equality constraints in (13).

\section{A. Existence of solutions to the finite LP}

We make the following assumption throughout this section.

Assumption 10: There exists $\theta^{a} \in \mathbb{R}^{N-1} \forall a=1, \ldots, M$, such that the LP in 13] is feasible for some $\gamma>1$. Note, Assumption 10 does not impose the requirement in 147. For the sake of simplicity and clarity of presentation, we will assume that the measure, $m$, in (13) is equivalent to the Lebesgue measure and $G>0$. Satisfaction of Assumption 10 can be verified using the following algorithm.

Algorithm 1 1) Set $\mathcal{I}:=1, \ldots, N-1, \mathcal{I}_{0}:=N, L=0$. 2) Set $\mathcal{I}_{L+1}:=\emptyset$. 3) For each $i \in \mathcal{I} \backslash\left\{\mathcal{I}_{0} \cup \ldots \cup \mathcal{I}_{L}\right\}$ do a) Pick the smallest $a \in 1, \ldots, M$ such that $\left(P_{T_{a}}\right)_{(i j)}>0$ for some $j \in \mathcal{I}_{L}$. b) If $a$ exists then, set $u_{N}\left(D_{i}\right):=u^{a}$, $\mathcal{I}_{L+1}:=\mathcal{I}_{L+1} \cup\{i\}$. 4) End For 5) If $\mathcal{I}_{0} \cup \ldots \cup \mathcal{I}_{L}=\mathcal{I}$ then, set $L=L+1$. STOP. 6) If $\mathcal{I}_{L+1}=\emptyset$ then, STOP. 7) Set $L=L+1$. Go to Step 2.

The algorithm iteratively adds to $\mathcal{I}_{L+1}$, set $D_{i}$, which has a non-zero probability of transition to any of the sets in $\mathcal{I}_{L}$. In graph theory terms, the above algorithm iteratively builds a tree starting with the set $D_{N} \supseteq \mathcal{A}$. If the algorithm terminates in Step 6, then we have identified sets $\mathcal{I} \backslash\left\{\mathcal{I}_{0} \cup \ldots \cup \mathcal{I}_{L}\right\}$ that cannot be stabilized with the controls in $\mathcal{U}_{M}$. If the algorithm terminates at Step 5, then we show in the Lemma below that a set of stabilizing controls exist.

Lemma 11: Let $\mathcal{X}_{N}=\left\{D_{1}, \ldots, D_{n}\right\}$ be a partition of the state space, $X$, and $\mathcal{U}_{M}=\left\{u^{1}, \ldots, u^{M}\right\}$ be a quantization of the control space, $U$. Suppose Algorithm 1 terminates in Step 5 after $L^{\text {max }}$ iterations, then the controls $u_{N}$ identified by the algorithm renders the system coarse stable.

Proof: Let $P_{T_{u_{N}}}$ represent the closed loop transition matrix resulting from the controls identified by Algorithm 1. Suppose $\mu \in \mathbb{R}^{N-1}, \mu \geq 0, \mu \neq 0$ be any initial distribution supported on the complement of the attractor set $\mathcal{X}_{N} \backslash D_{N}$. By construction, $\mu$ has a non-zero probability of entering the attractor set after $L^{\max }$ transitions. Hence,

$$
\sum_{i=1}^{N-1}\left(\mu^{\prime}\left(P_{T_{u_{N}}}^{1}\right)^{L^{\max }}\right)_{(i)}<\sum_{i=1}^{N-1}(\mu)_{(i)} \Longrightarrow \lim _{n \rightarrow \infty}\left(P_{T_{u_{N}}}^{1}\right)^{n L^{\max }} \longrightarrow 0 .
$$

Thus, the sub-Markov matrix $P_{T_{u_{N}}}^{1}$ is transient and implies the claim. 
Algorithm 1 is less expensive than the approaches proposed in [20], where a mixed integer LP and a nonlinear programming approach were proposed. The strength of our algorithm is that it is guaranteed to find deterministic stabilizing controls, if they exist. The following lemma shows an optimal solution to (13) exists under Assumption 10.

Lemma 12: Consider a partition $\mathcal{X}_{N}=\left\{D_{1}, \ldots, D_{N}\right\}$ of the state-space $X$ with attractor set $\mathcal{A} \subseteq D_{N}$ and a quantization $\mathcal{U}_{M}=\left\{u^{1}, \ldots, u^{M}\right\}$ of the control space $U$. Suppose Assumption 10 holds for some $\gamma>1$ and for $m, G>0$. Then, there exists an optimal solution, $\theta$, to the LP (13) and an optimal solution, $V$, to the dual LP (15) with equal objective values, $\left(\sum_{a=1}^{M}\left(G^{a}\right)^{\prime} \theta^{a}=m^{\prime} V\right)$ and $\theta, V$ bounded.

Proof: From Assumption [10, the LP (13) is feasible. Observe the dual LP in (15) is always feasible with a choice of $V=0$. The feasibility of primal and dual linear programs implies the claim as a result of LP strong duality [27].

Remark 13: Note, existence of an optimal solution does not impose a positivity requirement on the cost function, $G$. In fact, even assigning $G=0$ allows determination of a stabilizing control from the Lyapunov measure equation (13). In this case, any feasible solution to (13) suffices.

The next result shows the LP (13) always admits an optimal solution satisfying 14].

Lemma 14: Given a partition $\mathcal{X}_{N}=\left\{D_{1}, \ldots, D_{N}\right\}$ of the state-space, $X$, with attractor set, $\mathcal{A} \subseteq D_{N}$, and a quantization, $\mathcal{U}_{M}=\left\{u^{1}, \ldots, u^{M}\right\}$, of the control space, $U$. Suppose Assumption 10 holds for some $\gamma>1$ and for $m, G>0$. Then, there exists a solution $\theta \in \mathbb{R}^{N-1}$ solving (13) and $V \in \mathbb{R}^{N-1}$ solving 15 for any $\gamma \in\left[1, \bar{\gamma}_{N}\right)$. Further, the following hold at the solution: 1$)$ For each $j=1, \ldots,(N-1)$, there exists at least one $a_{j} \in 1, \ldots, M$, such that $(V)_{(j)}=\gamma\left(P_{T_{a_{j}}}^{1} V\right)_{(j)}+\left(G^{a_{j}}\right)_{(j)}$ and $\left(\theta^{a_{j}}\right)_{(j)}>0$. 2) There exists a $\tilde{\theta}$ that solves [13), such that for each $j=1, \ldots,(N-1)$, there is exactly one $a_{j} \in 1, \ldots, M$, such that $\left(\tilde{\theta}^{a_{j}}\right)_{(j)}>0$ and $\left(\tilde{\theta}^{a^{\prime}}\right)_{(j)}=0$ for $a^{\prime} \neq a_{j}$.

\section{Proof:}

From the assumptions, we have that Lemma 12 holds. Hence, there exists $\theta \in \mathbb{R}^{N-1}$ solving (13) and $V \in \mathbb{R}^{N-1}$ solving (15) for any $\gamma \in\left[1, \bar{\gamma}_{N}\right)$. Further, $\theta$ and $V$ satisfy following the first-order optimality conditions [27],

$$
\begin{gathered}
\sum_{a=1}^{M} \theta^{a}-\gamma \sum_{a=1}^{M}\left(P_{T_{a}}^{1}\right)^{\prime} \theta^{a}=m \\
V \leq \gamma P_{T_{a}}^{1} V+G^{a} \perp \theta^{a} \geq 0 \forall a=1, \ldots, M .
\end{gathered}
$$

We will prove each of the claims in order.

Claim 1: Suppose, there exists $j \in 1, \ldots,(N-1)$, such that $\left(\theta^{a}\right)_{(j)}=0$ for all $a=1, \ldots, M$. Substituting in the optimality conditions [16, one obtains,

$$
\gamma \sum_{a=1}^{M}\left(\left(P_{T_{a}}^{1}\right)^{\prime} \theta^{a}\right)_{(j)}=-(m)_{(j)}
$$

which cannot hold, since, $P_{T_{a}}^{1}$ has non-negative entries, $\gamma>0$ and $\theta^{a} \geq 0$. Hence, there exists at least one $a_{j}$ such that $\left(\theta^{a_{j}}\right)_{(j)}>0$. The complementarity condition in 16 then requires that $(V)_{(j)}=\left(\gamma P_{T_{a_{j}}}^{1} V\right)_{(j)}+\left(G^{a_{j}}\right)_{(j)}$. This proves the first claim. 
Claim 2: Denote $a(j)=\min \left\{a \mid\left(\theta^{a}\right)_{(j)}>0\right\}$ for each $j=1, \ldots,(N-1)$. The existence of $a(j)$ for each $j$ follows from statement 1 . Define $P_{T_{u}}^{1} \in \mathbb{R}^{(N-1) \times(N-1)}$ and $G^{u} \in \mathbb{R}^{N-1}$ as follows:

$$
\begin{aligned}
\left(P_{T_{u}}^{1}\right)_{(j i)} & :=\left(P_{T_{a(j)}}^{1}\right)_{(j i)} \forall i=1, \ldots,(N-1) \\
\left(G^{u}\right)_{(j)} & :=\left(G^{a(j)}\right)_{(j)}
\end{aligned}
$$

for all $j=1, \ldots,(N-1)$. From the definition of $P_{T_{u}}^{1}, G^{u}$ and the complementarity condition in [16, it is easily seen that $V$ satisfies

$$
V=\gamma P_{T_{u}}^{1} V+G^{u}=\lim _{n \rightarrow \infty}\left(\left(\gamma P_{T_{u}}^{1}\right)^{n} V+\sum_{k=0}^{n}\left(\gamma P_{T_{u}}^{1}\right)^{k} G^{u}\right) .
$$

Since $V$ is bounded and $G^{u}>0$, it follows that $\rho\left(P_{T_{u}}^{1}\right)<1 / \gamma$. Define $\tilde{\theta}$ as,

$$
\begin{gathered}
{\left[\begin{array}{c}
\left(\tilde{\theta}^{a(1)}\right)_{(1)} \\
\vdots \\
\left(\tilde{\theta}^{a(N-1)}\right)_{(N-1)}
\end{array}\right]=\left(I_{N-1}-\gamma\left(P_{T_{u}}^{1}\right)^{\prime}\right)^{-1} m} \\
\left(\tilde{\theta}^{a}\right)_{(j)}=0 \forall j=1, \ldots,(N-1), \quad a \neq a(j) .
\end{gathered}
$$

The above is well-defined, since we have already shown that $\rho\left(P_{T_{u}}^{1}\right)<1 / \gamma$.

From the construction of $\tilde{\theta}$, we have that for each $j$ there exists only one $a_{j}$, namely $a(j)$, for which $\left(\tilde{\theta}^{a(j)}\right)_{(j)}>0$. It remains to show that $\tilde{\theta}$ solves 13 . For this, observe

$$
\begin{aligned}
\sum_{a=1}^{M}\left(G^{a}\right)^{\prime} \tilde{\theta}^{a} & \stackrel{\text { 19b }}{=} \sum_{j=1}^{N-1}\left(G_{j}^{a(j)} \tilde{\theta}^{a(j)}\right)_{(j)} \stackrel{[19 \mathrm{a}]}{=}\left(G^{u}\right)^{\prime}\left(I_{N-1}-\gamma\left(P_{T_{u}}^{1}\right)^{\prime}\right)^{-1} m \\
= & \left(\left(I_{N-1}-\gamma P_{T_{u}}^{1}\right)^{-1} G^{u}\right)^{\prime} m \stackrel{18]}{=} V^{\prime} m .
\end{aligned}
$$

The primal and dual objectives are equal with the above definition of $\tilde{\theta}$. Hence, $\tilde{\theta}$ solves 13 . The claim is proved.

The following theorem states the main result.

Theorem 15: Consider a partition $\mathcal{X}_{N}=\left\{D_{1}, \ldots, D_{N}\right\}$ of the state-space, $X$, with attractor set, $\mathcal{A} \subseteq D_{N}$, and a quantization, $\mathcal{U}_{M}=\left\{u^{1}, \ldots, u^{M}\right\}$, of the control space, $U$. Suppose Assumption 10 holds for some $\gamma>1$ and for $m, G>0$. Then, the following statements hold: 1 ) there exists a bounded $\theta$, a solution to (13) and a bounded $V$, a solution to (15); 2) the optimal control for each set, $j=1, \ldots,(N-1)$, is given by $u\left(D_{j}\right)=u^{a(j)}$, where $a(j):=$ $\min \left\{a \mid\left(\theta^{a}\right)_{(j)}>0\right\}$; 3) $\mu$ satisfying $\gamma\left(P_{T_{u}}^{1}\right)^{\prime} \mu-\mu=-m$, where $\left(P_{T_{u}}^{1}\right)_{(j i)}=\left(P_{T_{a(j)}}^{1}\right)_{(j i)}$ is the Lyapunov measure for the controlled system.

Proof: Assumption 10 ensures that the linear programs 13 and 15 have a finite optimal solution (Lemma (12)). This proves the first claim of the theorem and also allows the applicability of Lemma 14 . The remaining claims follow as a consequence.

Although the results in this section assumed the measure, $m$, is equivalent to Lebesgue, this can be easily relaxed to the case where $m$ is absolutely continuous with respect to Lebesgue and is of interest where the system is not everywhere stabilizable. If it is known there are regions of the state-space not stabilizable, then $m$ can be chosen such that its support is zero on these regions. If the regions are not known a priori then, 13 can be modified to 


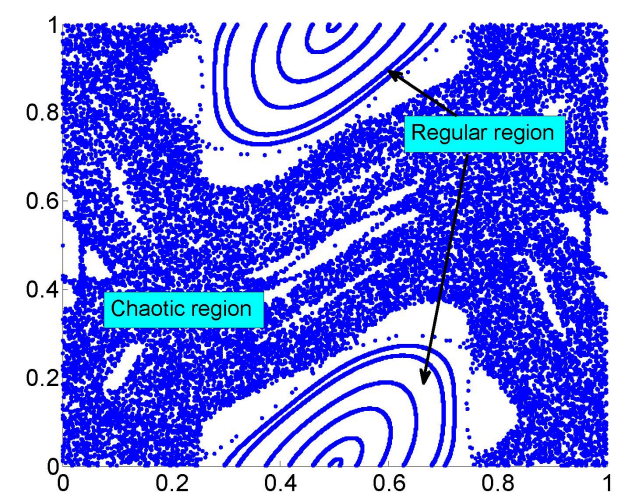

Fig. 1: Dynamics of the standard map.

minimize the $l_{1}$-norm of the constraint residuals. This is similar to the feasibility phase commonly employed in LP algorithms [28].

\section{EXAMPLES}

The results in this section have been obtained using an interior-point algorithm, IPOPT [29]. IPOPT is an opensource software available through the COIN-OR repository [30], developed for solving large-scale non-convex nonlinear programs. We solved the linear programs using the Mehrotra predictor-corrector algorithm [31] for linear and convex quadratic programs implemented in IPOPT.

\section{A. Standard Map}

The standard map is a 2D map also known as the Chirikov map. It is one of the most widely studied maps in dynamical systems [32].

The standard map is obtained by taking a Poincare map of one degree of freedom Hamiltonian system forced with a time-periodic perturbation and by writing the system in action-angle coordinates. The standard map forms a basic building block for studying higher degree of freedom Hamiltonian systems. The control standard map is described by the following set of equations [32]:

$$
\begin{gathered}
x_{n+1}=x_{n}+y_{n}+K u \sin 2 \pi x_{n}(\bmod 1) \\
y_{n+1}=y_{n}+K u \sin 2 \pi x_{n}(\bmod 1),
\end{gathered}
$$

where $(x, y) \in R:=\{[0,1) \times[0,1)\}$ and $u$ is the control input. The dynamics of the standard map with $u \equiv 1$ and $K=0.25$ is shown in Fig. 1. The phase space dynamics consists of a mix of chaotic and regular regions. With the increase in the perturbation parameter, $K$, the dynamics of the system become more chaotic.

For the uncontrolled system (i.e., $u \equiv 0$ ), the entire phase space is foliated with periodic and quasi-periodic orbits. In particular, every $y=$ constant line is invariant under the system dynamics, and consists of periodic and quasi-periodic orbits for rational and irrational values of $y$, respectively. Our objective is to globally stabilize 
the period two orbit located at $(x, y)=(0.25,0.5)$ and $(x, y)=(0.75,0.5)$, while minimizing the cost function $G(x, y, u)=x^{2}+y^{2}+u^{2}$. To construct the finite dimensional approximation of the P-F operator, the phase space, $R$, is partitioned into $50 \times 50$ squares: each cell has 10 sample points. The discretization for the control space is chosen to be equal to $u^{a}=[-0.5: 0.05: 0.5]$. In Figs. $2 \mathrm{a}$ and $2 \mathrm{p}$, we show the plot for optimal cost function and control input, respectively. We observe in Fig. $2 p$ the control values used to control the system are approximately antisymmetric about the origin. This antisymmetry is inherent in the standard map and can also be observed in the uncontrolled standard map plot in Fig. 2p.

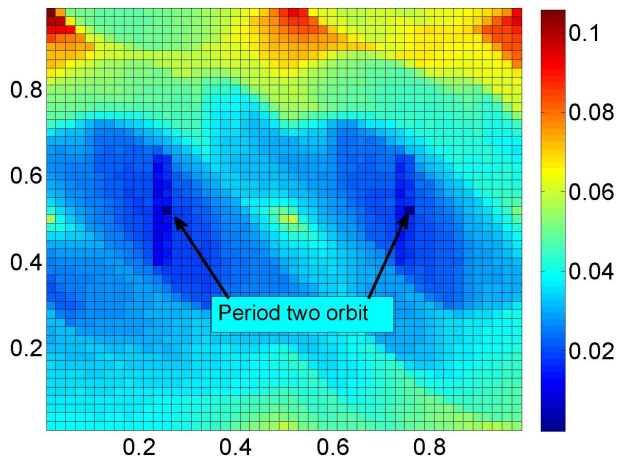

(a)

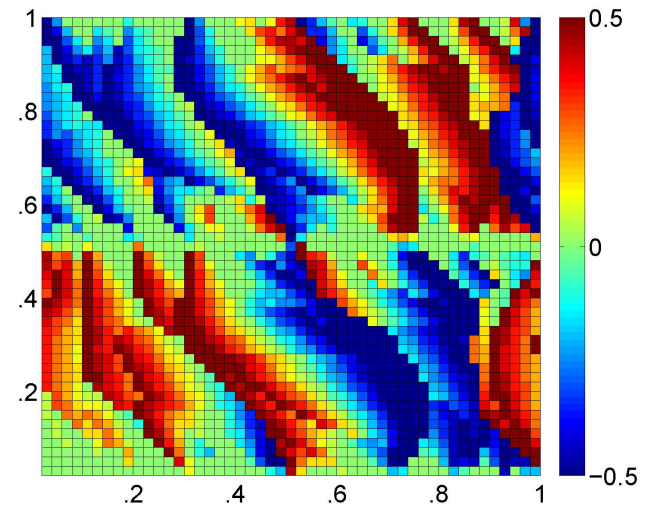

(b)

Fig. 2: a) Optimal cost function; b) Optimal control input.

\section{CONClusions}

Lyapunov measure is used for the optimal stabilization of an attractor set for a discrete time dynamical system. The optimal stabilization problem using a Lyapunov measure is posed as an infinite dimensional linear program. A computational framework based on set oriented numerical methods is proposed for the finite dimensional approximation of the linear program.

The set-theoretic notion of a.e. stability introduced by the Lyapunov measure offers several advantages for the problem of stabilization. First, the controller designed using the Lyapunov measure exploits the natural dynamics of the system by allowing the existence of unstable dynamics in the complement of the stabilized set. Second, the Lyapunov measure provides a systematic framework for the problem of stabilization and control of a system with complex non-equilibrium behavior.

\section{ACKNOWLEDGMENTS}

The authors would like to acknowledge Amit Diwadkar from Iowa State University for help with the simulation. We thank an anonymous referee of the previous version of the paper for improving the quality of the paper. This research work was supported by NSF Grant \# CMMI-0807666 and ECCS-1002053. 


\section{REFERENCES}

[1] R. B. Vinter, "Convex Duality and Nonlinear Optimal Control," SIAM J Control and Optimization, vol. 31, pp. 518-538, 1993.

[2] S. Hedlund and A. Rantzer, "Convex Dynamic Programming for Hybrid Systems," IEEE Transactions on Automatic Control, vol. 47, no. 9, pp. 1536-1540, 2002.

[3] S. Prajna and A. Rantzer, "Convex Programs for Temporal Verification of Nonlinear Dynamical Systems," SIAM Journal on Control and Optimization, vol. 46, no. 3, pp. 999-1021, 2007.

[4] R. Van Handel, "Almost global stochastic stability," SIAM Journal on Control and Optimization, vol. 45, pp. 1297-1313, 2006.

[5] O. Hernández-Lerma and J. B. Lasserre, Discrete-time Markov Control Processes: Basic Optimality Criteria. Springer-Verlag, New York, 1996.

[6] _ _ "Approximation schemes for infinite linear programs," SIAM J. Optimization, vol. 8, no. 4, pp. 973-988, 1998.

[7] L. Grüne, "Error estimation and adaptive discretization for the discrete stochastic Hamilton-Jacobi-Bellman equation," Numerische Mathematik, vol. 99, pp. 85-112, 2004.

[8] L. G. Crespo and J. Q. Sun, “Solution of fixed final state optimal control problem via simple cell mapping," Nonlinear dynamics, vol. 23, pp. 391-403, 2000.

[9] O. Junge and H. Osinga, "A set-oriented approach to global optimal control," ESAIM: Control, Optimisation and Calculus of Variations, vol. 10, no. 2, pp. 259-270, 2004.

[10] L. Grüne and O. Junge, "A set-oriented approach to optimal feedback stabilization," Systems Control Lett., vol. 54, no. 2, pp. 169-180, 2005.

[11] D. Hernandez-Hernandez, O. Hernandez-Lerma, and M. Taksar, "A linear programming approach to deterministic optimal control problems," Applicationes Mathematicae, vol. 24, no. 1, pp. 17-33, 1996.

[12] V. Gaitsgory and S. Rossomakhine, "Linear programming approach to deterministic long run average optimal control problems," SIAM J. Control ad Optimization, vol. 44, no. 6, pp. 2006-2037, 2006.

[13] J. Lasserre, C. Prieur, and D. Henrion, "Nonlinear optimal control: Numerical approximation via moment and LMI-relaxations," in Proceeding of IEEE Conference on Decision and Control, Seville, Spain, 2005.

[14] S. Meyn, "Algorithm for optimization and stabilization of controlled Markov chains," Sadhana, vol. 24, pp. 339-367, 1999.

[15] M. Bardi and I. Capuzzo-Dolcetta, Optimal control and viscosity solutions of Hamilton-Jacobi-Bellman equations. Boston: Birkhauser, 1997.

[16] A. Rantzer, "A dual to Lyapunov's stability theorem," Systems \& Control Letters, vol. 42, pp. 161-168, 2001.

[17] C. Prieur and L. Praly, "Uniting local and global controller," in Proceedings of IEEE Conference on Decision and Control, AZ, 1999, pp. 1214-1219.

[18] U. Vaidya and P. G. Mehta, "Lyapunov measure for almost everywhere stability," IEEE Transactions on Automatic Control, vol. 53, pp. 307-323, 2008.

[19] R. Rajaram, U. Vaidya, M. Fardad, and B. Ganapathysubramanian, "Almost everywhere stability: Linear transfer operator approach," Journal of Mathematical analysis and applications, vol. 368, pp. 144-156, 2010.

[20] U. Vaidya, P. Mehta, and U. Shanbhag, "Nonlinear stabilization via control Lyapunov measure," IEEE Transactions on Automatic Control, vol. 55, pp. 1314-1328, 2010.

[21] A. Raghunathan and U. Vaidya, "Optimal stabilization using Lyapunov measures," http://www.ece.iastate.edu/ ugvaidya/publications.html, 2012.

[22] —_ "Optimal stabilization using Lyapunov measure," in Proceedings of American Control Conference, Seattle, WA, 2008, pp. $1746-1751$.

[23] A. Lasota and M. C. Mackey, Chaos, Fractals, and Noise: Stochastic Aspects of Dynamics. New York: Springer-Verlag, 1994.

[24] U. Vaidya, "Converse theorem for almost everywhere stability using Lyapunov measure," in Proceedings of American Control Conference, New York, NY, 2007.

[25] H. Furstenberg, Recurrence in Ergodic theory and Combinatorial Number Theory. Princeston, New Jersey: Princeston University Press, 1981.

[26] E. Anderson and P. Nash, Linear Programming in Infinite-Dimensional Spaces - Theory and Applications. John Wiley \& Sons, Chichester, U.K., 1987. 
[27] O. L. Mangasarian, Nonlinear programming, ser. Classics in Applied Mathematics. Philadelphia, PA: Society for Industrial and Applied Mathematics (SIAM), 1994, vol. 10, corrected reprint of the 1969 original.

[28] S. J. Wright, Primal-Dual Interior-Point Methods. Philadelphia, Pa: Society for Industrial and Applied Mathematics, 1997.

[29] A. Wächter and L. T. Biegler, "On the implementaion of a primal-dual interior point filter line search algorithm for large-scale nonlinear programming," Mathematical Programming, vol. 106, no. 1, pp. 25-57, 2006.

[30] COIN-OR Repository. [Online]. Available: http://www.coin-or.org/|

[31] J. Nocedal and S. Wright, Numerical optimization, ser. Springer series in operations research. Springer, 2006. [Online]. Available: http://books.google.com/books?id=eNlPAAAAMAAJ

[32] U. Vaidya and I. Mezić, “Controllability for a class of area preserving twist maps,” Physica D, vol. 189, pp. 234-246, 2004. 